\title{
A LC-MS/MS METHOD FOR THE QUANTIFICATION OF DEFLAZACORT METABOLITE IN HUMAN PLASMA: DEVELOPMENT, VALIDATION AND APPLICATION TO A PHARMACOKINETIC STUDY
}

\author{
*Sarita Karthikeyan ${ }^{1,2}$ Anju Aji ${ }^{1,2}$, Sarabjit Singh ${ }^{2}$, Shivanand Puthli ${ }^{2}$ \\ ${ }^{1}$ JJT University, Jhunjhunu, Rajasthan, India \\ ${ }^{2}$ Panacea Biotec Ltd, 72/3, TTC Industrial Area, Mahape, Navi Mumbai, Maharashtra, India \\ *Corresponding author's email: saritakarthikeyan@panaceabiotec.com
}

\begin{abstract}
Deflazacort is a methyl-oxazoline derivative of prednisolone used for its anti-inflammatory and immunosuppressive effects. It is an inactive prodrug which is converted rapidly to its active metabolite 21-desacetyl deflazacort. A simple, rapid, sensitive and specific liquid chromatography-tandem mass spectrometry method was developed for the estimation of 21-desacetyl deflazacort in human plasma. Sample preparation involved simple solid phase extraction of 21-desacetyl deflazacort and internal standard. The samples were analyzed using a reversed phase column and detected using positive mode ESI tandem mass spectrometry. The LLOQ was $0.5 \mathrm{ng} / \mathrm{mL}$ and the assay was linear over the range of $0.5-100 \mathrm{ng} / \mathrm{mL}$ using a sample volume of $250 \mu \mathrm{L}$. The method was validated for specificity, linearity, precision, accuracy and stability parameters. The method was applied to the analysis of plasma samples after oral administration of $6 \mathrm{mg}$ DFZ tablets of marketed product in healthy adult subjects in a fasting bioavailability study.
\end{abstract}

Keywords: LC-MS/MS, deflazacort, bioanalytical, validation

\section{INTRODUCTION}

Deflazacort (DFZ) is a glucocorticoid prescribed as an anti-inflammatory and immunosuppressant. It is a synthetic oxazoline derivative of prednisolone, having the molecular formula11beta, 21-dihydroxy-2'-methyl-5'betaH-pregna-1, 4-dieno [17,16d] oxazole-3, 20-dione-21acetate $^{1}$. DFZ administered orally is well-absorbed and converted by plasma esterases to pharmacologically active metabolite 21-desacetyl deflazacort (21-DFZ), which achieves peak plasma concentration in 1.5-2 hours. Its elimination plasma half-life is $1.1-1.9$ hours. It is $40 \%$ protein-bound and has no affinity for corticosteroid binding globulin (transcortin). Elimination takes place primarily through the kidneys (70\%). The remaining 30\% is eliminated in the faeces ${ }^{2}$.

Previously reported methods for determination of 21-DFZ include detection by high performance liquid chromatography $^{3}$ and liquid chromatography-mass spectrometry (LC-MS) ${ }^{4,5,6}$. The reported method based on LC-MS detection used the relatively tedious technique of liquid-liquid extraction (LLE) for analyte extraction from plasma $4,5,6$. The other reported method used C18cartridge based solid phase extraction (SPE) extraction followed by semi-microbore liquid chromatography for determination of 21-DFZ using a high sample volume of $2 \mathrm{ml}$ human plasma $^{3}$. The reported method lacks the selectivity and sensitivity offered by liquid chromatography-tandem mass spectrometry (LC-MS/MS) for high-throughput analysis of 21-DFZ. Quantification of drugs in biological matrices by LCMS is becoming more common due to the improved sensitivity and specificity of this technique 7 . SPE methods have better extraction efficiency, reproducibility and they yield cleaner extracts compared to $\mathrm{LLE}^{8}$. The polymer-based SPE cartridges provide greater analyte retention and overcome many limitations of the silica-based cartridges ${ }^{9}$.
The primary objective was to develop and validate a rapid, sensitive and specific LC-MS/MS method for 21-DFZ estimation in human plasma. We applied this method to samples of a PK study of DFZ in 26 healthy volunteers after oral administration of $6 \mathrm{mg}$ DFZ tablets of commercial product.

\section{EXPERIMENTAL}

\section{Chemicals and Reagents}

21-DFZ (Figure 1) was procured from Toronto Research Chemicals Inc, Canada and Internal Standard (IS) prednisolone (Figure 1) was obtained from Sigma Aldrich, Germany. Acetonitrile (HPLC gradient grade), methanol (LCMS grade) and formic acid (puriss grade) were purchased from Sigma Aldrich, USA. Purified water was produced using Milli Q Gradient Millipore system (Millipore, USA). Drug free human plasma containing K3 EDTA as anticoagulant was used as the blank matrix.

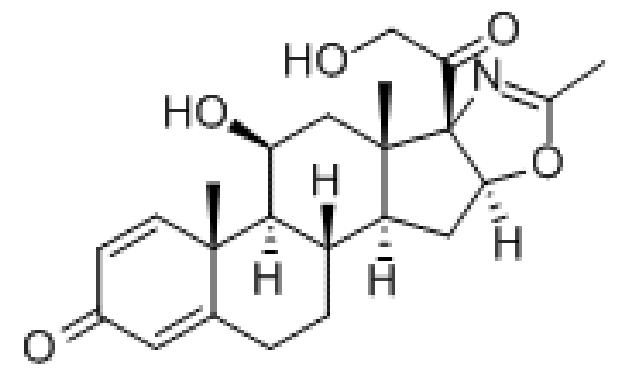

Figure 1(A): Chemical Structures of 21-desacetyl deflazacort 
<smiles>C[C@]12C=CC(=O)C=C1CC[C@@H]1[C@@H]2CC[C@]1(C)C(=O)CO</smiles>

B

Figure 1(B): Chemical Structures of IS (prednisolone).

\section{Instrumentation and chromatographic conditions}

Chromatographic separation was achieved by using Waters Acquity BEH C18 column having dimensions of $50 * 2.1 \mathrm{~mm}$ using a Waters Acquity UPLC system (Waters, USA). Formic acid (0.1\%): Acetonitrile (20:80, v/v) was used as the mobile phase at a flow rate of $0.3 \mathrm{~mL} / \mathrm{min}$. The column temperature was set at $40^{\circ} \mathrm{C}$ and the sample compartment temperature was set at $5^{\circ} \mathrm{C}$. $5 \mu \mathrm{L}$ of the sample was injected and the MS detection was performed using a Quattro Premier XE Mass spectrometer (Waters, USA) with an electrospray source. The desolvation temperature and source temperature were set at $400^{\circ} \mathrm{C}$ and $110^{\circ} \mathrm{C}$ respectively. The electrospray source was operated in the positive ionization mode and $\mathrm{m} / \mathrm{z} 400.06 \rightarrow 123.91$ and $361.36 \rightarrow 146.96$ were monitored for quantitation of 21-DFZ and prednisolone respectively. Data acquisition was performed with MassLynx software (Version 4.1, Waters, USA).

\section{Preparation of standards and Quality Control (QC) samples}

Stock solutions of 21-DFZ and prednisolone were prepared in methanol $(1 \mathrm{mg} / \mathrm{mL})$. Further dilutions of 21DFZ to make substocks were done using diluent (water: methanol 50:50) to obtain solutions of $0.0125 \mu \mathrm{g} / \mathrm{mL}$, $0.025 \mu \mathrm{g} / \mathrm{mL}, 0.150 \mu \mathrm{g} / \mathrm{mL}, 0.500 \mu \mathrm{g} / \mathrm{mL}, 1.000 \mu \mathrm{g} / \mathrm{mL}$, $1.60 \mu \mathrm{g} / \mathrm{mL}, 2.000 \mu \mathrm{g} / \mathrm{mL}$ and $2.50 \mu \mathrm{g} / \mathrm{mL}$. Four levels of QCs (low, medium2 and high) were also prepared at concentrations of $0.0375 \mu \mathrm{g} / \mathrm{mL}, 1.20 \mu \mathrm{g} / \mathrm{mL}$ and 1.8 $\mu \mathrm{g} / \mathrm{mL}$.

The calibration curve standards were prepared by spiking the substocks in blank plasma to obtain concentration of $0.50 \mathrm{ng} / \mathrm{mL}, \quad 1.00 \mathrm{ng} / \mathrm{mL}, \quad 6.0 \mathrm{ng} / \mathrm{mL}, \quad 20.0 \mathrm{ng} / \mathrm{mL}, \quad 40.0$ $\mathrm{ng} / \mathrm{mL}, 64.00 \mathrm{ng} / \mathrm{mL}, 80.00 \mathrm{ng} / \mathrm{mL}$ and $100.00 \mathrm{ng} / \mathrm{mL}$ respectively.

The QC concentrations of $1.50 \mathrm{ng} / \mathrm{mL}, 48.0 \mathrm{ng} / \mathrm{mL}$ and $72.00 \mathrm{ng} / \mathrm{mL}$ were prepared by spiking appropriate $21-\mathrm{DFZ}$ QC substock solutions in blank human plasma.

\section{Sample preparation}

The plasma samples stored at $-80^{\circ} \mathrm{C}$ were thawed at room temperature before processing. Ten microlitres of internal standard solution (prednisolone $5.0 \mu \mathrm{g} / \mathrm{mL}$ ) were added to $250 \mu \mathrm{L}$ of sample aliquoted in a centrifuge tube and mixed for 10 seconds. The sample was then loaded on the SPE cartridge previously conditioned with $1 \mathrm{ml}$ of methanol and equilibrated with $1 \mathrm{ml}$ of water. The cartridge was washed with $1 \mathrm{ml}$ of water and $0.5 \mathrm{ml}$ of $50 \%$ methanol in water. Elution was carried out using $0.5 \mathrm{ml}$ of methanol:acetonitrile(1:1). The elute was evaporated to dryness under a nitrogen stream at $40^{\circ} \mathrm{C}$. The residue was dissolved in $150 \mu \mathrm{L}$ of acetonitrile: water $(80: 20)$, transferred to a 96 well plate and injected into chromatographic system.

\section{Method Validation procedure}

The method was validated according to the recent US Food and Drug Administration (FDA) guidelines ${ }^{10}$. Specificity, linearity, lower limit of quantification (LLOQ), inter-day and intra-day precision and accuracy as well as recovery and stability of 21-DFZ were evaluated. The matrix effect and dilution integrity were also studied.

\section{Specificity}

The specificity of the method was determined by screening six different batches of blank human plasma. Blank and LLOQ level standards were prepared from each of the six lots as per the sample preparation method described earlier.

\section{Sensitivity}

Six samples at the LLOQ level were processed as per procedure and injected. The $\% \mathrm{CV}$ and the \% accuracy were calculated.

\section{Linearity/Calibration curve}

The calibration curves were constructed using values generated from the calibration standards by plotting the analyte to internal standard peak area ratios against concentration. The suitability of the curves was confirmed by back-calculating the concentrations of the calibration standards.

\section{Intra-day and intraday precision and accuracy (P\&A)}

The intra-day precision and accuracy was performed by processing six samples at each QC level and analyzing against the calibration curve. The $\% \mathrm{CV}$ and \% accuracy at each QC level was calculated. For inter-day precision and accuracy, \% CV and \% accuracy of the QC samples at each of the five levels (LLOQ, LQC, MQC and HQC) of two consecutive $\mathrm{P} \& \mathrm{~A}$ batches on different days were calculated.

\section{Recovery}

Aqueous solutions at the four QC levels were prepared. Recovery of analyte and internal standard from plasma was calculated by comparing the area response of analyte and drug in the extracted sample to the area response of the analyte and drug in the neat solution at the same concentrations.

\section{Matrix effect}

The matrix effect was evaluated by comparing the peak area ratios of analyte to IS in the post-extraction spiked blank plasma samples to the peak area ratios obtained by injecting neat standards directly. The matrix factor (MF) was calculated for all six samples at LQC and HQC levels and the matrix effect was determined by (MF-1) x100.

\section{Reinjection reproducibility}

The QC samples at low QC and high QC levels of the precision and accuracy batch were reinjected after a gap of 8 hours and analyzed against previously analysed calibration curve. The $\%$ change of the initially obtained 
Sarita et al

Journal of Drug Delivery \& Therapeutics; 2013, 3(2), 75-82

QC concentrations and those obtained after reinjection were calculated.

\section{Dilution integrity}

The effect of a 1:5 dilution on the determination of 21DFZ in human plasma was determined by measuring five samples of spiked DQC (dilution quality control) in human plasma. The DQC concentration (204.624 ng/mL) was two times the ULOQ concentration. The spiked samples were diluted 1:5 with drug free human plasma and then processed. The QC sample concentrations were calculated using appropriate dilution factor.

\section{Stability}

The stability of stock solutions of 21-DFZ and prednisolone were evaluated after storage for 36days at $20^{\circ} \mathrm{C}$. Stability of 21-DFZ in human plasma was assessed by analyzing 21-DFZ samples at two concentrations, low QC and high QC after exposing them to different conditions of time and temperature. The bench top stability was evaluated after exposure of the plasma samples at room temperature for 4.5 hours. The freeze thaw stability was determined after three freeze-thaw cycles from $-80^{\circ} \mathrm{C}$ to room temperature at regular intervals. The samples were frozen at $-80^{\circ} \mathrm{C}$ for 24 hours and thawed at room temperature. After complete thawing, the samples were refrozen at $-80^{\circ} \mathrm{C}$ for $12-24 \mathrm{hrs}$ at $-80^{\circ} \mathrm{C}$. This step was repeated two times. After the third thawing stage, the samples were analyzed.

The long-term stability was assessed after storage of the test samples at $-80^{\circ} \mathrm{C}$ and then analyzed after 42days of storage.

The post-preparative storage stability of 21-DFZ was assessed by the autosampler stability and dried extract stability. The processed samples in vials were stored in the autosampler for 25 hours and then reinjected and analyzed against fresh calibration curve.
For dried extract stability, the samples were processed till the evaporation step; the dried extracts were stored at $80^{\circ} \mathrm{C}$ for $24 \mathrm{hrs}$. After $24 \mathrm{hrs}$, the frozen dried extracts were retrieved from the $-80^{\circ} \mathrm{C}$, thawed, reconstituted and analyzed against fresh calibration curve.

\section{Clinical Study}

The study protocol was approved by independent ethics committee and the study was conducted as per pertinent requirements of the current ICMR guidelines and USFDA adopted ICH guidelines for Good Clinical Practice and Declaration of Helsinki (Seoul 2008). An open label bioavailability investigation study in 26 healthy, adult, human subjects under fasting condition was conducted. One tablet of $6 \mathrm{mg}$ DFZ were administered to the subjects along with $240 \mathrm{ml}$ of water and blood samples were collected at $0.50,1.00,1.25,1.5,1.75,2.00,2.25,2.5$, $3.00,4.00,6.00,8.00,10.00,12.00,16.00,20.00,22.00$, 24.00 , and $36.00 \mathrm{hrs}$.

\section{RESULTS AND DISCUSSION}

\section{Method development: MS and chromatographic development}

The MS tuning parameters and MS/MS fragmentation were optimized for both 21-DFZ and IS by varying the cone voltage and collision energy. Neat solutions of $0.1 \mu \mathrm{g} / \mathrm{ml}$ concentration were infused at a pump flow rate of $10 \mu \mathrm{l} / \mathrm{min}$ in the ESI positive ion mode. The peak in the mass spectrum at $\mathrm{m} / \mathrm{z} 400.06$ corresponded to the protonated molecule $(\mathrm{M}+\mathrm{H}){ }^{+}$of 21-DFZ. The most abundant daughter ion fragment at $\mathrm{m} / \mathrm{z} 123.91$ in the product ion spectrum was selected to obtain MRM transition to gain maximum sensitivity. Further, an MRM transition for prednisolone was also optimized under the same conditions.

Full scan and product ion spectra of 21-desacetyl deflazacort and prednisolone are shown in Figure 2.

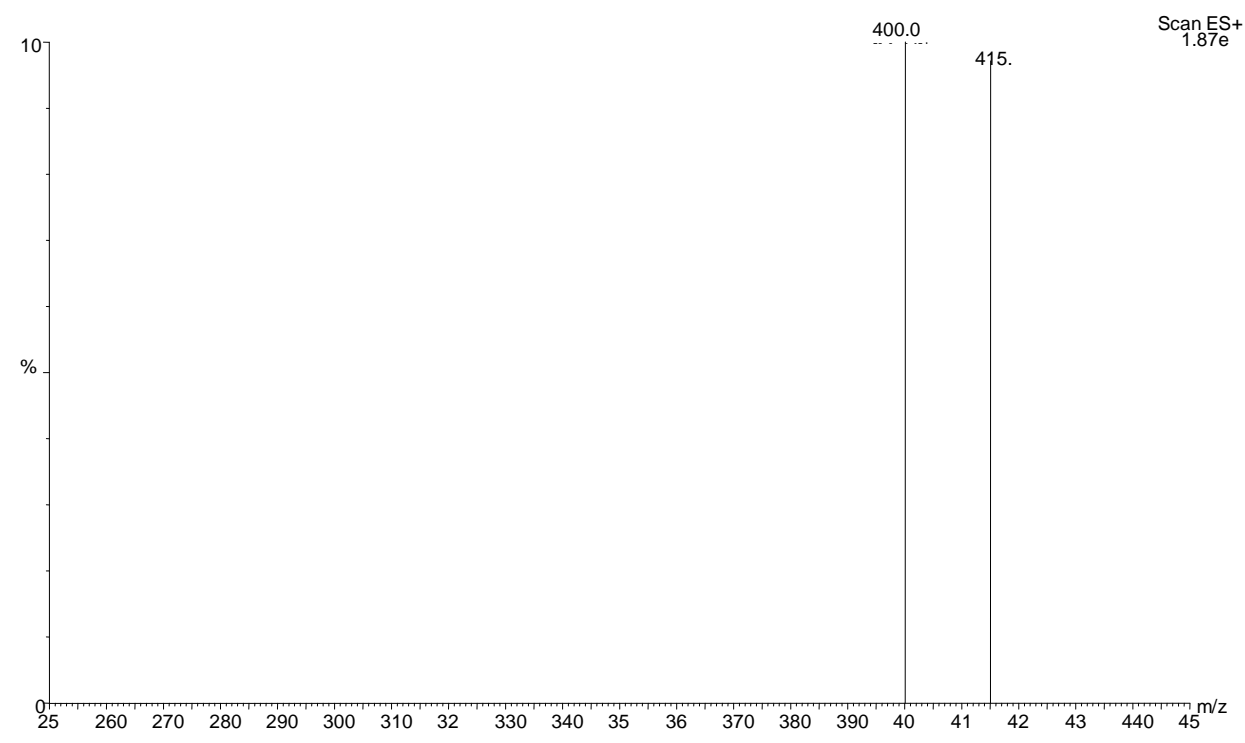

Figure 2(A): full scan spectra of 21-DFZ protonated molecule 


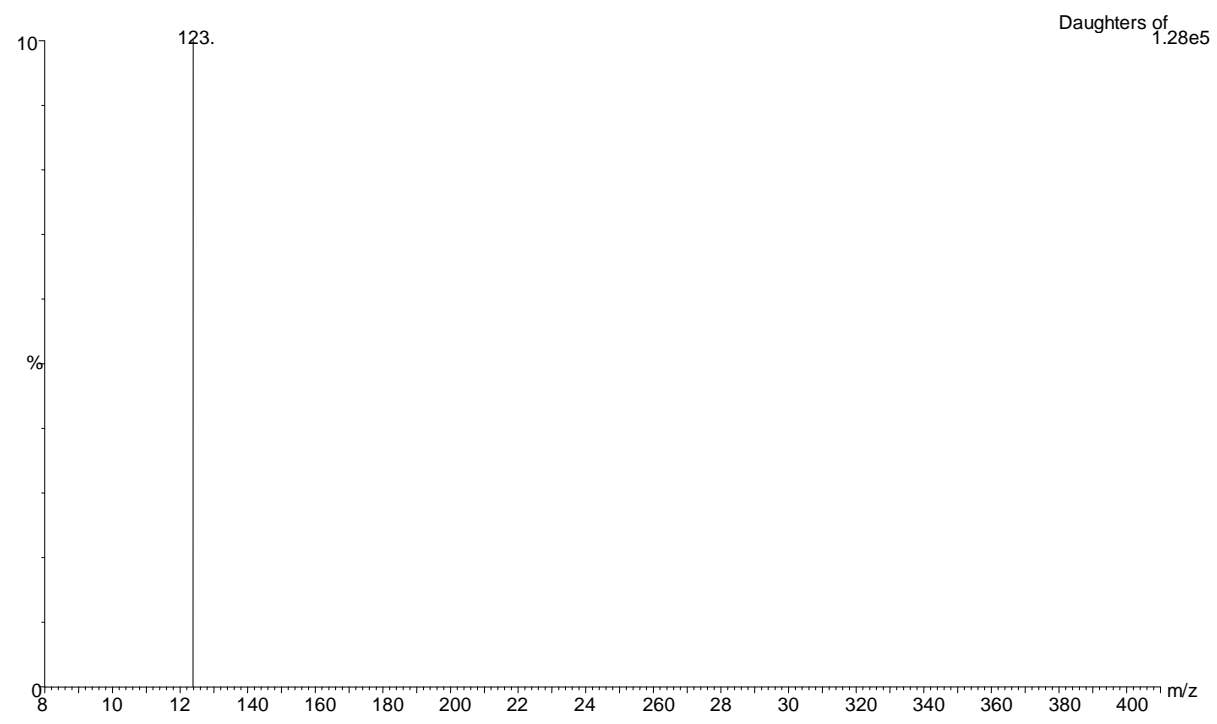

Figure 2(B): product ion spectra of 21-DFZ

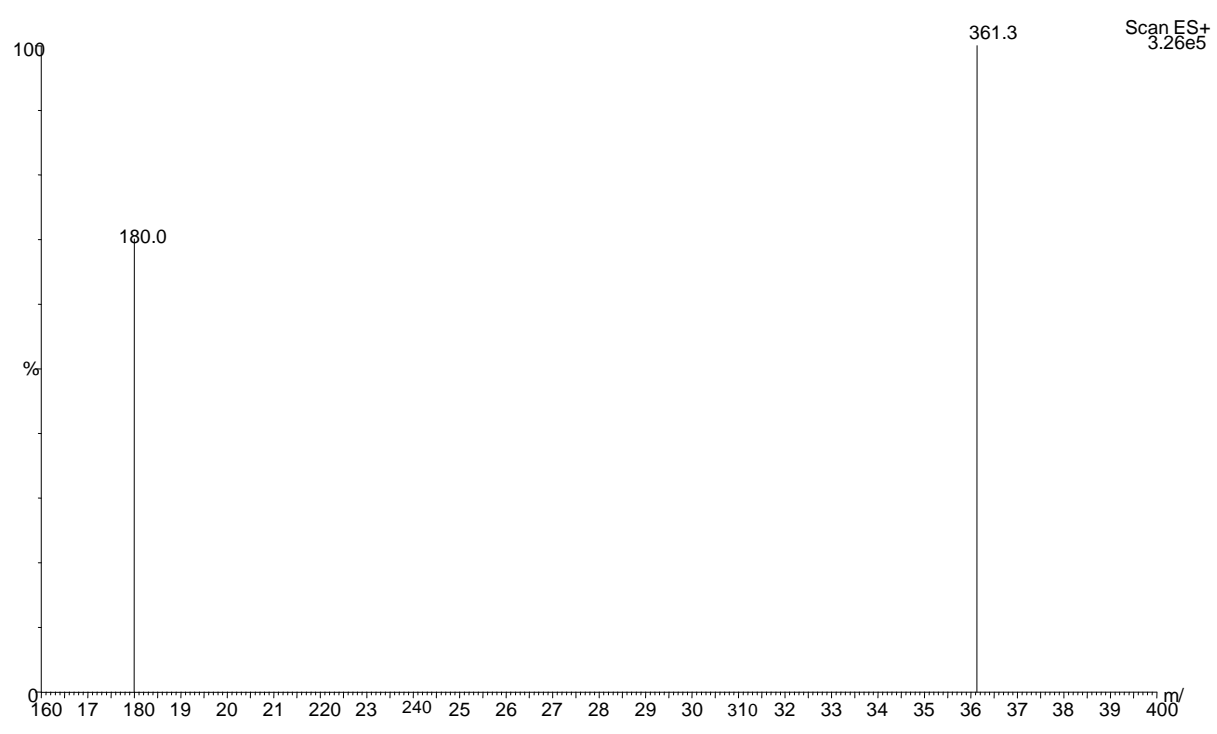

Figure 2(C): full scan spectra of IS

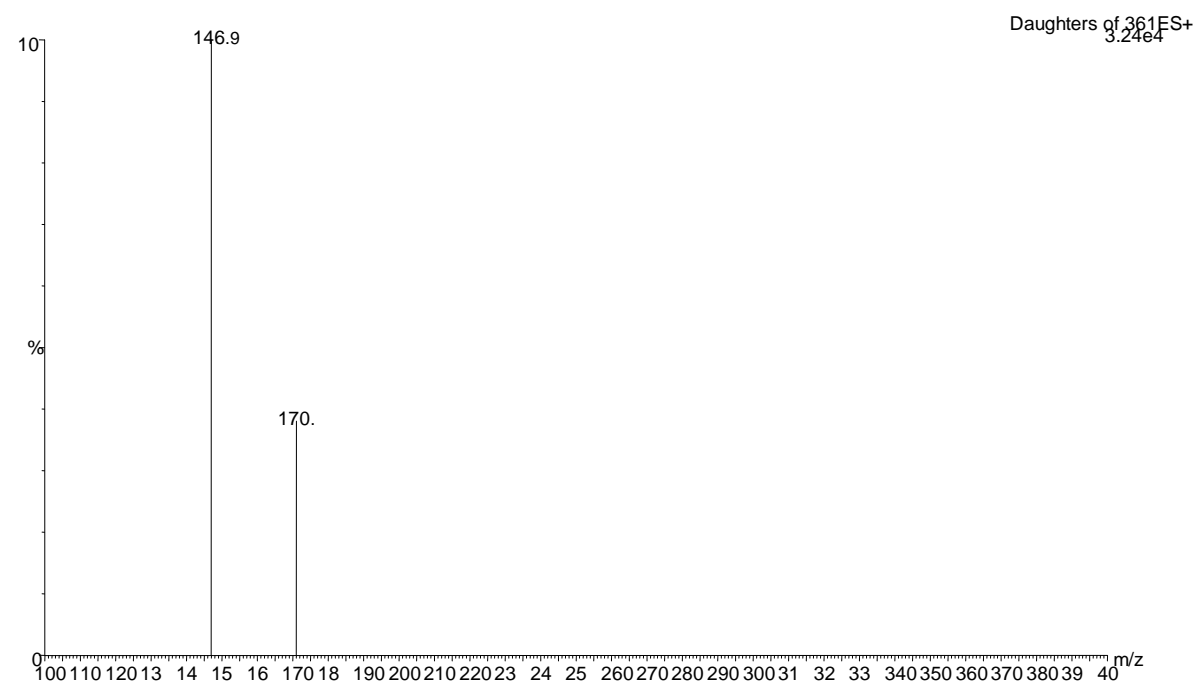

Figure 2(D): product ion spectra of IS.

Prednisolone was chosen as a suitable internal standard as it is structurally related to 21-DFZ. Various compositions of acetonitrile and formic acid $(0.1 \%)$ were investigated with a view to optimize the mobile phase for sensitivity, speed and peak shape. Acetonitrile and formic acid $(0.1 \%)$ with a composition of 80:20 (v/v) gave better chromatographic results and was selected as the mobile phase. The retention time of both 21-DFZ and internal 
Sarita et al

standard was $0.45 \mathrm{~min}$. Both polymer-based and monolithic LC columns were assessed for their suitability for the chromatographic separation of the analyte. A relatively short run time $(2.0 \mathrm{~min})$ was achieved with the short sub- 2 micron column and a high organic content in the mobile phase. The typical chromatograms are shown in Figure 3.

(A)

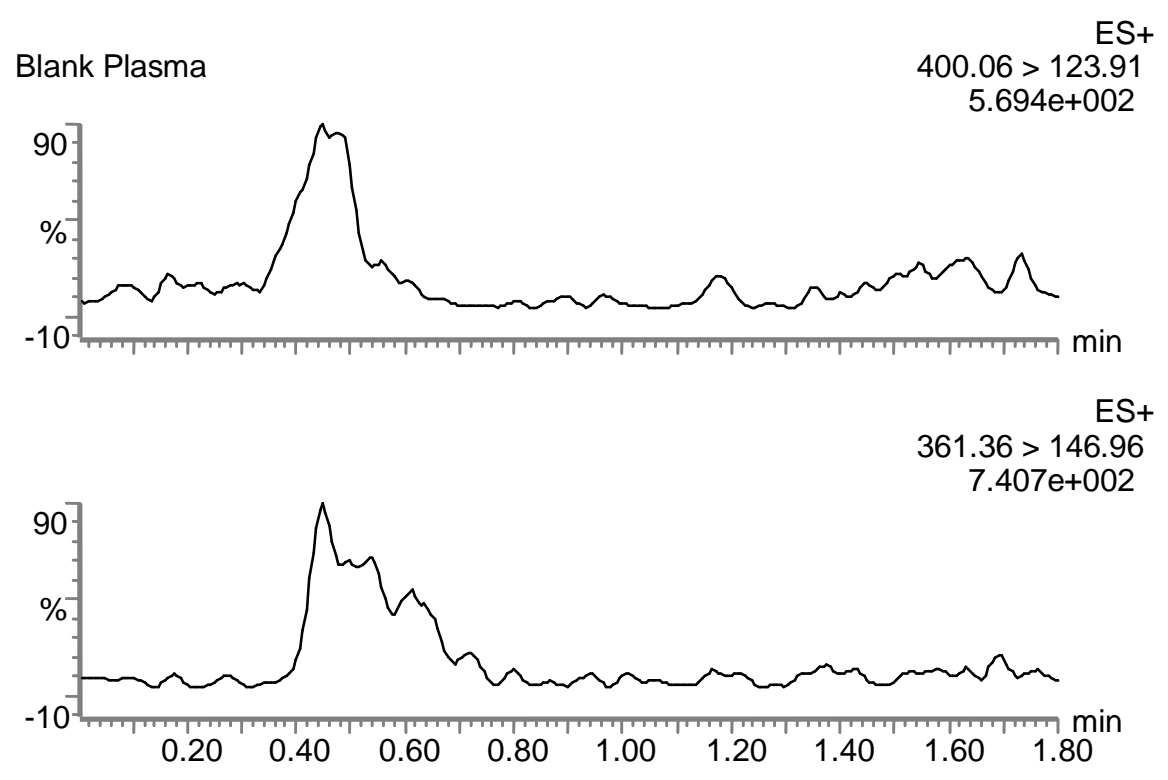

(B)

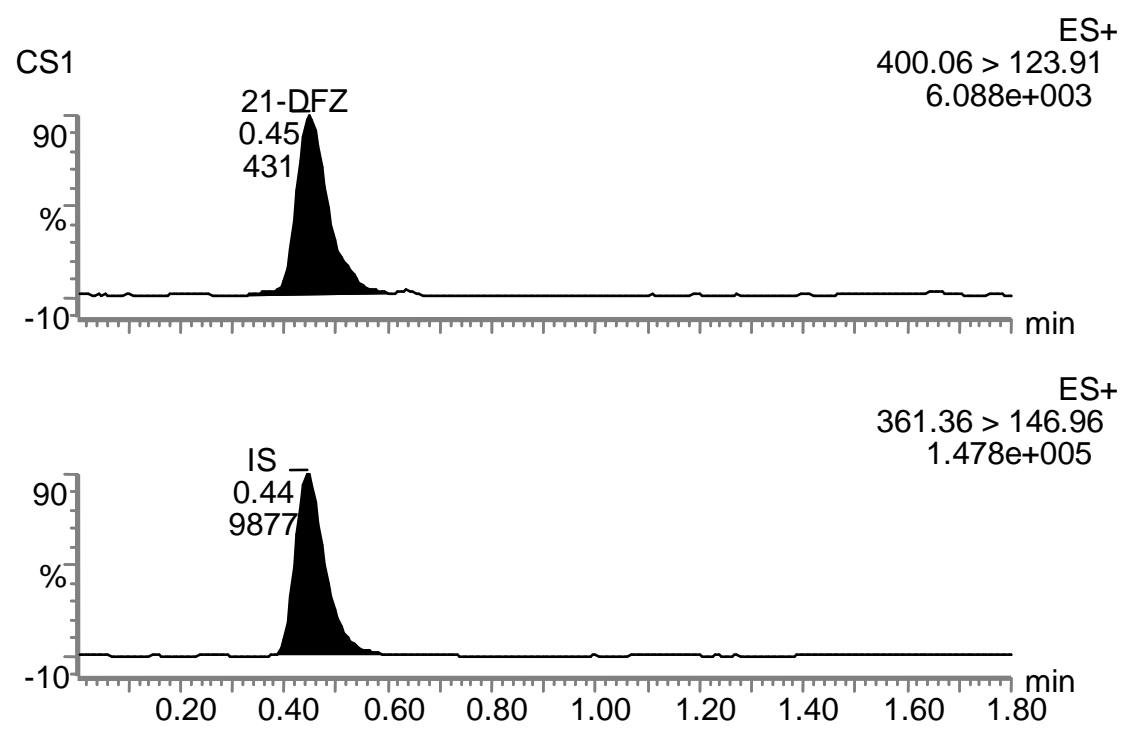

Figure 3: Representative chromatograms of (A) blank plasma sample and (B) plasma sample spiked with 21desacetyl deflazacort at lower limit of quantification (LLOQ).

\section{Method development: Sample preparation}

Various sample preparation trials were carried out by LLE using several compositions of tertiary butyl methyl ether and diethyl ether: ethyl acetate. SPE methods were also evaluated with several modifications in washing \& elution steps. SPE using polymer-sorbent cartridges gave reasonably good recovery without any blank interference. The SPE technique was chosen as the sample processing method over LLE for its consistent recovery, reproducibility and speed of analysis.

\section{Method Validation}

Selectivity
All the six lots of tested plasma had no significant interfering peaks at the retention time of analyte or internal standards. All six lots met the acceptance criteria.

\section{Sensitivity}

The mean of the six samples prepared at the LLOQ concentration level was $95.3 \%$ with a CV of $4.0 \%$

\section{Linearity}

The linear regression was fitted over the concentration range of 0.5 to $100 \mathrm{ng} / \mathrm{mL}$ of 21-DFZ in human plasma by a $1 / x$ weighted least squares linear regression. For the four consecutive batches, the calibration curve standards showed an overall accuracy of $89.82-107.90 \%$ with RSD 
Sarita et al

Journal of Drug Delivery \& Therapeutics; 2013, 3(2), 75-82

of less than $14 \%$. The results are shown in Table 1 . Good linearity was obtained in the validated concentration range. The correlation coefficients of the $1 / \mathrm{x}$ weighted calibration curves from consecutive batches were in the range of 0.997-0.999. A typical calibration curve is shown in Figure 4.

Table 1: Precision and accuracy of calibration standards of 21-desacetyl deflazacort in human plasma from three consecutive validation batches

\begin{tabular}{|c|c|c|c|}
\hline $\begin{array}{c}\text { Nominal calibration standard } \\
\text { concentration }(\mathrm{ng} / \mathrm{ml})\end{array}$ & $\begin{array}{c}\text { Mean Back-calculated calibration standard } \\
\text { concentration (ng/ml) }\end{array}$ & $\%$ CV Accuracy \\
\hline 0.506 & 0.455 & 11.6 & 89.96 \\
\hline 1.011 & 0.980 & 14.0 & 96.97 \\
\hline 6.068 & 6.547 & 5.8 & 107.90 \\
\hline 20.227 & 21.572 & 0.4 & 106.65 \\
\hline 40.454 & 43.410 & 3.9 & 107.31 \\
\hline 64.727 & 58.138 & 1.9 & 89.82 \\
\hline 80.909 & 79.592 & 1.4 & 98.37 \\
\hline 101.136 & 104.344 & 103.17 \\
\hline
\end{tabular}

Compound name: 21-DFZ

Correlation coefficient: $r=0.999107, r^{\wedge} 2=0.998215$

Calibration curve: $0.067114{ }^{*} \mathrm{x}+0.00503812$

Curve type: Linear, Origin: Exclude, Weighting: $1 / x$

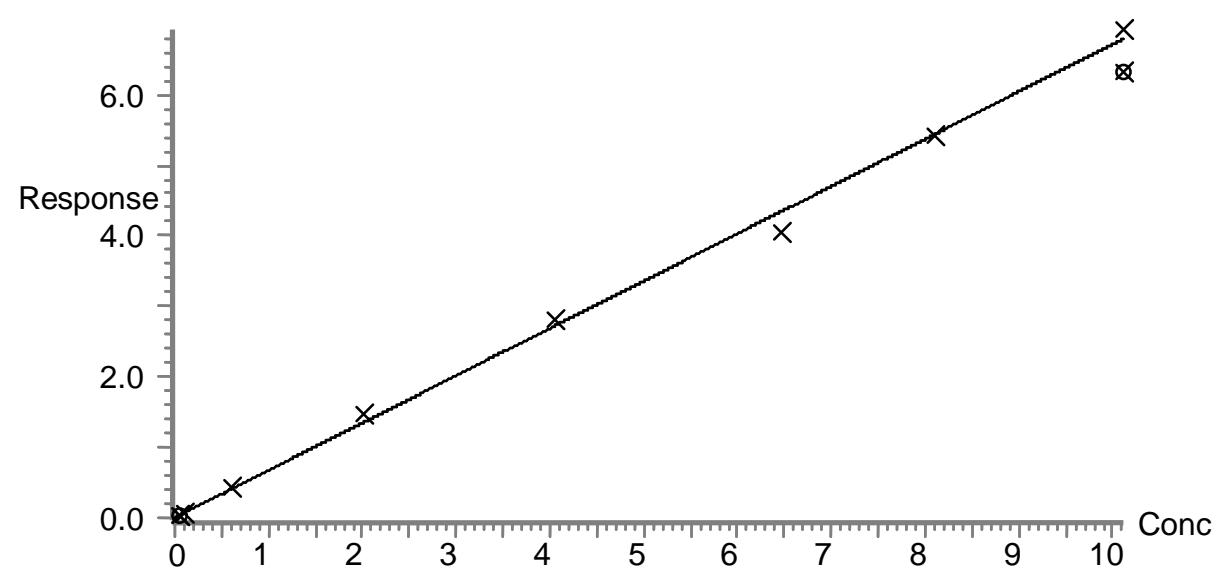

Figure 4: Representative Calibration Curve for the validated range of 0.5 to $100 \mathrm{ng} / \mathrm{ml}$

\section{Intra-day and inter-day precision and accuracy}

The resulting intra and inter-day precision and accuracy data for each spiked QC concentration are presented in Table 2. These results indicate that the method is reliable and reproducible within its analytical range.

Table 2: Intraday and Interday Precision and accuracy of QC samples for 21-desacetyl deflazacort in human plasma

\begin{tabular}{|c|c|c|c|c|c|c|c|}
\hline \multirow[t]{2}{*}{ QC levels } & \multirow{2}{*}{$\begin{array}{c}\text { Nominal QC } \\
\text { concentration } \\
(\mathrm{ng} / \mathrm{ml})\end{array}$} & \multicolumn{3}{|c|}{ Intraday Precision and accuracy $(n=6)$} & \multicolumn{3}{|c|}{ Interday Precision and accuracy $(n=16)$} \\
\hline & & $\begin{array}{c}\text { Mean } \\
(\mathrm{ng} / \mathrm{ml})\end{array}$ & $\% \mathrm{CV}$ & \%accuracy & $\begin{array}{c}\text { Mean } \\
(\mathrm{ng} / \mathrm{ml})\end{array}$ & $\% \mathrm{CV}$ & \%accuracy \\
\hline LLOQ & 0.512 & 0.488 & 4.01 & 95.3 & 0.505 & 9.36 & 98.6 \\
\hline LQC & 1.535 & 1.364 & 6.76 & 88.9 & 1.424 & 10.85 & 92.8 \\
\hline MQC & 49.110 & 45.482 & 5.14 & 92.6 & 46.657 & 4.75 & 95.0 \\
\hline HQC & 73.665 & 70.441 & 6.73 & 95.6 & 72.904 & 7.22 & 99.0 \\
\hline
\end{tabular}

\section{Recovery}

The recovery of $21-\mathrm{DFZ}$ was $80.50 \%, 84.59 \%$ and $92.36 \%$ at the low QC, medium QC and high QC levels respectively. The mean global recovery of 21-DFZ across the three levels of QCs was $85.82 \pm 7.01 \%$. The results showed that 21-DFZ had consistent recovery across all four levels. The mean recovery of the internal standard was $76.94 \%$.

\section{Matrix effect}

(c) 2011, JDDT. All Rights Reserved
The mean matrix effect for both LQC and HQC concentrations was $-3.35 \%$. The result indicated that the samples were sufficiently cleaned up in the extraction procedure resulting in minimal matrix effect in the method.

\section{Re-injection reproducibility}

The mean $\%$ change in obtained QC concentrations after initial analysis and after reinjection was $-1.56 \%$ at the LQC level, $-5.48 \%$ at the MQC level and $7.02 \%$ at the 
HQC level. The method met the acceptance criteria for reinjection reproducibility after $8 \mathrm{hrs}$.

\section{Dilution Integrity}

The six DQC samples processed after 1/5 dilution had an accuracy of $113.83 \%$ with a CV of $0.59 \%$. The dilution integrity at $1 / 5$ dilution met the acceptance criteria.

\section{Stability}

Acceptable stability was indicated in stock solution after storage at $-20^{\circ} \mathrm{C}$ for 36 days for $21-\mathrm{DFZ}$ and internal standard. Stability of 21-DFZ in plasma was demonstrated at bench top conditions for 4.5 hours and over three freeze/thaw cycles. 21-DFZ was found to be stable in plasma after long term storage at $-80^{\circ} \mathrm{C}$ for 42 days. The processed samples in the autosampler were stable for 25 hrs at $5^{\circ} \mathrm{C}$ whereas the processed samples at the dry extract stage were stable at $-80^{\circ} \mathrm{C}$ for $24 \mathrm{hrs}$. No significant degradation of 21-DFZ was observed in any of the stability test conditions and the results were consistent. The results are summarized in Table 3.

Table 3: Summary of stability data of 21-desacetyl deflazacort in human plasma

\begin{tabular}{|c|c|c|c|c|}
\hline Stability condition & Stability duration & QC Level & Mean Accuracy & $\begin{array}{c}\text { Mean Precision } \\
(\% \mathrm{CV})\end{array}$ \\
\hline \multirow{2}{*}{ Bench top } & \multirow{2}{*}{ 4.5hrs } & LQC & 101.5 & 8.42 \\
\cline { 3 - 5 } & & HQC & 93.2 & 11.83 \\
\hline \multirow{2}{*}{ Freeze thaw } & \multirow{2}{*}{3 cycles } & LQC & 101.22 & 10.44 \\
\cline { 3 - 5 } & & HQC & 99.40 & 7.04 \\
\hline \multirow{2}{*}{ Long term } & \multirow{2}{*}{42 days } & LQC & 107.28 & 12.61 \\
\cline { 3 - 5 } & & HQC & 89.714 & 6.92 \\
\hline \multirow{2}{*}{ Autosampler } & \multirow{2}{*}{$25 \mathrm{hrs}$} & LQC & 102.8 & 4.28 \\
\cline { 3 - 5 } & & HQC & 97.55 & 5.89 \\
\hline \multirow{2}{*}{ Dried Extract } & \multirow{2}{*}{$24 \mathrm{hrs}$} & LQC & 93.72 & 12.83 \\
\cline { 3 - 5 } & & HQC & 95.70 & 9.15 \\
\hline
\end{tabular}

\section{Clinical application}

The above validated LC-MS/MS method was successfully used to estimate pharmacokinetic parameters for 21-DFZ in human plasma. A mean plot of 21-DFZ concentration in plasma versus time was plotted from the data generated as shown in Figure 5. The pharmacokinetic parameters were calculated using Winnonlin software (Version 5.3 Pharsight, USA). The mean values were Cmax 38.19 ng/ml, Tmax $1.00 \mathrm{hrs}$ and AUC $(0 \rightarrow \mathrm{t}) 120.04 \mathrm{ng} . \mathrm{hr} / \mathrm{mL}$.

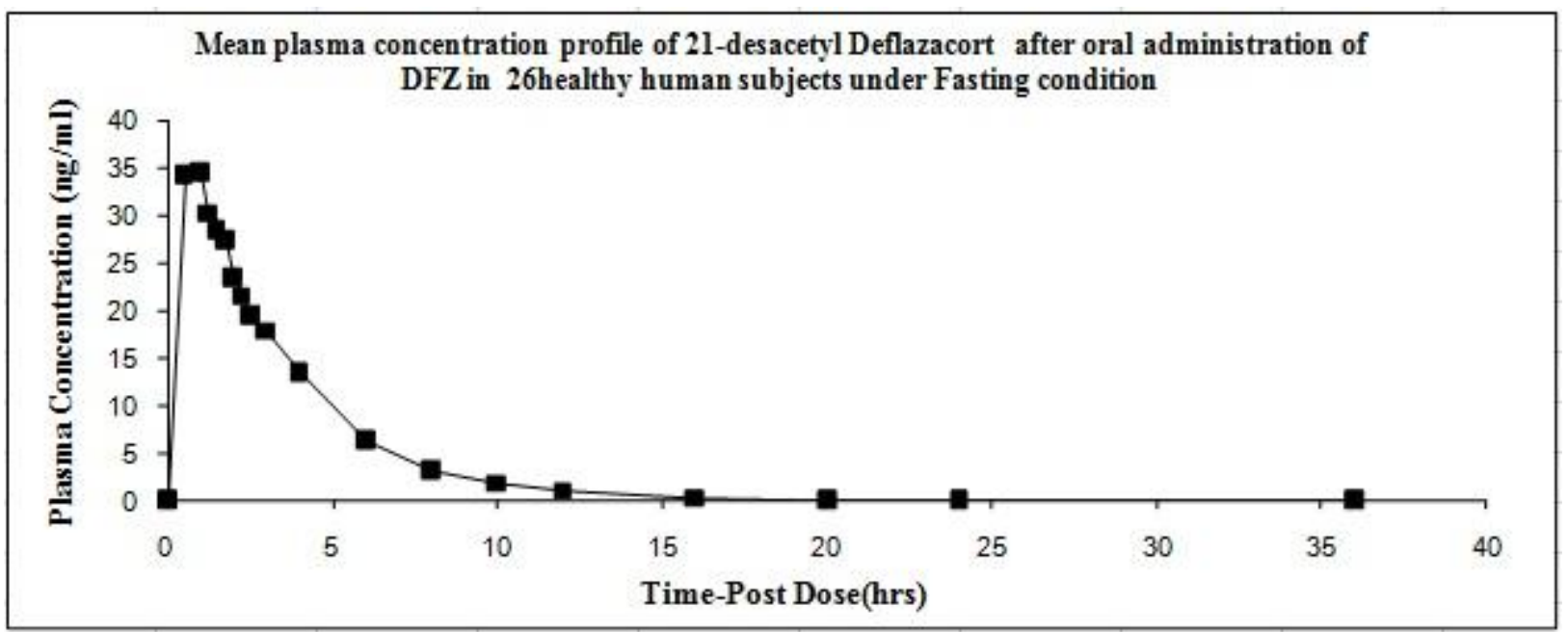

Figure 5: Mean Plasma concentration-time profile of 21-DFZ following administration of 1x6mg deflazacort formulation to healthy subjects in fasting condition $(n=26)$.

\section{CONCLUSION}

A simple, rapid and sensitive LC-MS/MS method for the determination of 21-desacetyl Deflazacort in human plasma was developed. The method allows high sample through-put due to its short run time and relatively simple sample preparation procedure. This method is reliable,

convenient and meets the criteria for application in clinical pharmacokinetic studies.

\section{ACKNOWLEDGMENT}

The authors wish to thank the management of Panacea Biotec Ltd for providing permission to publish this work. 


\section{REFERENCES}

1. Surajit N and Basanti A, Deflazacort Versus other Glucocorticoids: A comparison, Indian J of Dermatology, 2008, 53(4), 167-170.

2. Sunil RP, Gurudutt NU, Harsha KP, Deflazacort, Asian Journal of Ear, Nose and Throat, 2011, 9-12.

3. Reynolds DL, Burmaster SD, Eichmeier LS, Quantitative determination of 21-hydroxy-deflazacort in human plasma using gradient semi-microbore liquid chromatography, Biomed Chromatogr, 1994, 8(5),230-5.

4. Muralidharan S, Subramania NM, Determination of deflazacort in human plasma by liquid chromatography-mass spectrometry after liquid liquid extraction and its application in human pharmacokinetic studies, Pharmaceutical methods, 2011,2(2), 106-111.

5. Karina R, Oscar A, Nohemy O, Oscar G, Determination of 21desacetyl deflazacort in human plasma by LS-MS/MS assay, 2009 AAPS Annual Meeting and Exposition,2009.

6. (Ifa DR, Moraes ME, Moraes MO, Santagada V, Caliendo G, de Nucci G, Determination of 21-hydroxydeflazacort in human plasma by high-performance liquid chromatography/atmospheric pressure chemical ionization tandem mass spectrometry. Application to bioequivalence study, J Mass Spectrom., 2000, 35(3), 440-5.

7. Vita M, Skansen P, Hassan M, Abdel-Rehim M, Development and validation of a liquid chromatography and tandem mass spectrometry method for determination of roscovitine in plasma and urine samples utilizing online sample preparation. J.Chromatogr B, 2005, 817:303-307.

8. Nair A, Sharma D, Mittal R, Gupta N, Singh K, Quantitative Bioanalysis by LC-MS/MS: A Review, Journal of Pharmaceutical and Biomedical Sciences, 2010,7(7),1-9

9. Zwir-Ference A, Bizuik M, Solid Phase Extraction Technique: Trends, Opportunities and Applications, Polish Journal of Environ. Stud., 2006, 15(5), 677-690.

10. Guidance for Industry US-FDA Bioanalytical Method Validation, Center for Drug Evaluation and Research. 2001, http://www.fda.gov/cder/guidance/index.html 